\title{
JAHRESTAGUNG 1976 DER ARBEITSGEMEINSCHAFT DEUTSCHE LATEINAMERIKA-FORSCHUNG (ADLAF)
}

\author{
Von Gerhard Scheffler
}

Die Jahrestagung 1976 der ADLAF am 16./17. Juni in Hamburg, ausgerichtet vom Institut für Iberoamerika-Kunde im Verbund der Stiftung Deutsches Übersee-Institut, begleitete ein wissenschaftliches Kolloquium zum Thema „Gegenwartsprobleme Mexikos aus historischer, sozio-kultureller, politischer und ökonomischer Sicht“" unter Teilnahme von insgesamt knapp 90 Wissenschaftlern aus den ibero-amerikanischen Forschungsstellen des deutschsprachigen Raumes wie auch aus Mexiko selbst. Den Auftakt bildete der temparamentvolle Vortrag von Leopoldo Zea, Universidad Nacional Autónoma de México, der sich weitausholend mit den beiden Begriffen „Latinoamérica y el Tercer Mundo“ auseinandersetzte, Begriffen, die nur allzuoft reflexionslos in der Entwicklungsländerdiskussion verwandt werden. "Lateinamerika" umschreibt dabei zweifellos die amerikanischen Länder in ihrer gemeinsamen historischen, sprachlichen und damit kulturellen sowie religiösen Wurzel aus der spanisch-portugiesisch-französischen Kolonialzeit, wobei die karibischen Staaten mit britischer und niederländischer Vergangenheit mit eingeschlossen werden müssen. Es waren Franzosen (Tisserand, Chevalier, Domenech), die Anfang der sechziger Jahre des vorigen Jahrhunderts zur Zeit Napoleons III, die Latinität und den Panlatinismus als Gegenkraft zum Panslawismus und zum Yankeetum vertraten. Und wiederum waren es Franzosen (Sauvy und Balandier), die zur Zeit de Gaulles den Begriff „Dritte Welt“ gegenüber dem Kapitalismus und dem Kommunismus gebrauchten, um Frankreich eine Einflußmöglichkeit zwischen diesen großen Kräften zu erhalten. Bereits aus diesem historischen Kontext heraus handelt es sich demnach um Begriffe, die durchaus als imperialistisch zu begreifen sind (S. 2/3 und 7)1. Insofern entbehrt es nicht einer gewissen Ironie, daß diese Begriffe von den Entwicklungsländern aufgenommen werden, um ihre eigene Identität hinreichend zu beschreiben, und auch aufgenommen werden müssen, um der simplifizierenden Alternative Kapitalismus - Kommunismus zu entgehen.

Johanna Broda, z. Z. am Instituto Nacional de Antropología e Historia in Mexiko tätig, forderte mit ihrem Vortrag „La sociedad indigena de México después de la Conquista: Continuidad y cambio de estructuras socioculturales“ eindringlich, die indianischen Elemente in der mexikanischen Sozial- und Kulturgeschichte nicht immer nur getrennt nach den vor- und nachkolumbianischen Perioden darzustellen: Die Geschichte der indianischen Bevölkerung vor und unter den spanischen Eroberern ist nämlich nicht nur als singuläres Ereignis zu werten, sondern ebenso als Beispiel von Eroberung und Fremdherrschaft, Überschichtung von kulturell und ethnisch verschiedener Bevölkerungen, sie bedingenden sowie daraus resultierenden Dominationsmechanismen, sozio-kultureller Adaption unterdrückter Bevölkerungsgruppen, Widerstand und Konformismus, Kollaboration und Rebellion. Eine derartige Sicht läßt die Mexikanistik Anschluß finden an eine allgemeine sozialwissenschaftliche und historische Problematik.

1 Zitiert nach dem hektographierten Redemanuskript. 
Ein streng wirtschaftshistorisch bestimmtes Thema bot Wolfgang Müller, Universität Bonn, anhand der „Probleme der mexikanischen Industrialisierung während des Porfiriats". Die Einbettung des Industriesektors in die allgemeine Wirtschaft Mexikos zu Beginn unseres Jahrhunderts gerann dabei zu einem abschließenden Zahlenwerk.

Hans Werner Tobler, Universität Zürich, gab unter dem Titel „Agrarfrage, Bauernaufstände und politisch-gesellschaftliche Stabilisierung Mexikos während der Revolution 1910-1940" einen Beitrag zu einem hinreichend bekannten Thema, das mit der dazu vorhandenen mexikanischen und US-amerikanischen Literatur wahrlich ganze Bibliotheken füllt. Wenn es Tobler auf Grund seiner profunden Kenntnisse eben dieser Literatur gleichwohl gelang, diesem Thema wenn nicht neue, so doch verlorenzugehen drohende Gesichtspunkte abzugewinnen, so deshalb, weil er kritische Anmerkungen anzubringen gewillt war: Er verwies auf eine differenzierte Betrachtungsweise für die vorrevolutionäre Hacienda und auf die regional verschiedene Beteiligung der Landbewohner an den Aufstandsbewegungen, so daß insgesamt die Stärke und der Umfang der agrarrevolutionären Komponente in der mexikanischen Revolution $\mathrm{zu}$ relativieren sind ${ }^{2}$. Aufschlußreich ist danach auch, daß etwa im Staate Veracruz die Bauern „von oben“ organisiert wurden, um einer Reformpolitik überhaupt die Massenbasis zu geben ${ }^{3}$.

Nahezu nahtlos schloß sich der politologische Vortrag von Manfred Mols, Universität Mainz, an, der die heute relevanten „Faktoren der politischen Stabilität Mexikos" nachzeichnete: $\mathrm{Zu}$ einem ersten Faktorenbündel gehört der Einsatz von „pan y garrote", einem breiten Fächer der politischen Korrumpierungsmöglichkeiten ${ }^{4}$. Die zweite Faktorengruppe beruht auf dem jahrzehntelangen ökonomischen Wachstum des postrevolutionären Mexikos, das sowohl die vertikale wie die horizontale Mobilität großer Teile der Bevölkerung erleichtert und damit zur politischen Stabilität beiträgt. Dieses Wachstum ermöglichte für Individuen und Gruppen mit einer erhöhten ökonomischen, sozialen und politischen Energie die persönliche bzw. gruppeneigene Promotion. Als dritte Gruppe erscheinen bei Mols alle Faktoren, die die nicht zu leugnende Effizienz der herrschenden Machtelite im Sinne materieller politischer Leistungen begründen, allerdings unter gleichzeitigem Hinweis darauf, daß es bei Durchsicht aller tatsächlichen oder potentiellen Oppositionsgruppen eine umfassende Alternative zum derzeitigen System nicht gibt $^{5}$. Die vierte Faktorengruppe umfaßt die internationale Verflechtung Mexikos, insbesondere mit den USA. Die wechselseitige außenpolitische Ruicksichtnahme mit ihrer stabilisierenden Wirkung für das „Erfolge“ vorzeigende Regime funktioniert seit Jahrzehnten, wenn auch die Grenzen der von den USA tolerierten mexikanischen Inkonformität sichtbar werden ${ }^{6}$.

2 Hektographiertes Redemanuskript S. 9.

3 Redemanuskript S. 11.

4 Z. B. für den Bereich der Gewerkschaftsführer von Domitra, Michael: Die Rolle der Gewerkschaften im mexikanischen Herrschaftssystem, Bonn-Bad Godesberg 1975, geschildert.

5 Das Unvermögen der einzigen landesweit durchorganisierten und mit eigenem Vorfeld versehenen Oppositionsparte, der PAN - Partido de Acción Nacional -, zeigte sich etwa darin, daß sie zu den Präsidentschaftswahlen vom 4. 7. 1976 wegen interner Streitigkeiten keinen Kandidaten aufzustellen vermochte, El Heraldo de México v. 23. 2. 1976.

6 Aus jüngster Zeit sei verwiesen auf die schwankende Haltung Mexikos in der UN-Zionismusdebatte, die letztlich zum Rücktritt von Außenminister Rabasa Ende 1975 führte; auf die Einführung der 200-sm-Wirtschaftszone (dazu Scheffler, in: VRU 3/1976, S. 373) erst, nachdem die USA ihren Widerstand im Verlaufe der III. UN-Seerechtskonferenz aufgegeben hatte, und auf die. Warnung vor dem Beitritt Mexikos zur OPEC. 
Am Vorabend der Präsidentschafts- und Kongreßwahlen fanden die Ausführungen von Luis J. Molina Piñeiro, Mexiko, zu den vorhergehenden Wahlen 1973 ein aktuelles Interesse. Da bis 1962 alle Abgeordneten der Deputiertenkammer direkt in den Wahldistrikten mit Mehrheit gewählt wurde, schlug sich die Vorherrschaft der Partido Revolucionario Institucional - PRI - in erdrückenden Mehrheitsverhältnissen nieder: Allein die PAN vermochte als eigenständige Oppositionspartei noch nennenswerte Abgeordnetenzahlen zu erringen: $1961^{7}:$ PRI $=172$, PAN $=5$ Sitze.

Unter der Präsidentschaft von Lopez Mateos wurde daher unter Abänderung von Art. 54 der Verfassung mit dem Wahlgesetz von $1962^{8}$ das Institut des „diputado de partido “ eingeführt für diejenigen registrierten Parteien, die wenigstens 2,5\% der Stimmen erringen und in diesem Falle wenigstens 5 Deputierte zuzüglich je einen Deputierten pro weitere 0,5\% Stimmenanteil bis maximal 20 Abgeordnete zugeteilt erhalten. Diese Wahlgesetzänderung schlug sich daraufhin in folgender Sitzverteilung nieder:

1964: $\mathrm{PRI}=175$, PAN $=20$, andere Parteien $=15$ Sitze. $\mathrm{Da}$ jedoch die kleinen Parteien nicht einmal mehr 2,5\% erreichten ${ }^{10}$, wurde durch das Wahlgesetz vom 5. Januar 1973 das Quorum auf 1,5\% gesenkt ${ }^{11}$. Diese Wahlergebnisse ${ }^{12}$ beruhen nicht auf eindeutigen Wahlfälschungen, mögen sie im Einzelfall auch nicht ausgeschlossen sein ${ }^{13}$, wohl aber weitgehend auf der die Wahlentscheidung der Wähler in den weniger entwickelten ländlichen Regionen manipulierenden Einflußnahme der miteinander verfilzten Verwaltungs-, Regierungs-, Gewerkschafts-, Partei- und Bauernfunktionärsschicht. Dementsprechend kann sich kraft der größeren politischen Mobilität der wachsenden Mittelschichten in den urbanen Regionen ein ausgeglicheneres Wahlergebnis niederschlagen: So erzielte die PAN in einer der größten Städte, in Puebla, 59,5\% und in México, D. F., immerhin 39,3\%14. 1976 gewann die PRI 194 der 196 Direktmandate, die PAN erhielt 23 diputados de partido, die PPS 9 diputados de partido und die PARM davon 8 zuzüglich zweier Direktmandate ${ }^{15}$.

Unter teilweiser inhaltlicher Ubberlappung mit den Ausführungen von Mols stellte Wolf Grabendorff, Ebenhausen, die „innenpolitische Funktion der mexikanischen Außenpolitik“ unter Echeverría seit 1971 dar. Drei Veränderungen zum Nachteil Mexikos waren Anlaß zur verstärkten außenpolitischen Aktivität16: Das absehbare Ende des milagro mexicano, die protektionistische Außenhandelspolitik der USA und die größer werdenden innenpolitischen Schwierigkeiten. Grabendorff zeichnete vier Etappen in der "neuen" Außenpolitik nach (wobei seine punktuelle Analyse Kritik unter den Tagungsteilnehmern hervorrief): (1) Der Ausbau der Beziehungen zu Lateinamerika, insbesondere zum Chile Allendes ${ }^{17}$, unter Beto-

7 Nach Furtak, Robert K.: Revolutionspartei und politische Stabilität in México, Hamburg 1969, S. 65.

8 D. O. v. 22. 6. 1963.

9 Furtak a. a. O. S. 65; Meyer, Lorenzo, in: Comercio Exterior 1975, S. 1334 ff. (1335).

101970 erreichte die PARM - Partido Auténtico de la Revolución Mexicana - 0,81\% und die PPS Partido Popular Socialista $-1,35 \%$

11 Im einzelnen vgl. Brambila, Antonio, in: línea No. 20 (März/April 1976), S. 37 ff. (46/7).

121973 erhielt die PRI mit 69,55\% von 194 Direktmandaten 189; von den anderen Parteien erhielten mit Hilfe des deputado de partido die PAN 25, PPS 10 und PARM 6 Sitze; Länderbericht Mexiko, in: Wirtschaftsbericht-Lateinamerika der Deutschen Bank AG, Juli 1976, S. 27.

13 „Auffallende“ Wahlergebnisse auch 1973 etwa in Chetumal und Comitán, wo die PRI offiziell 98,2\% und 98,6 \% erreichte, Meyer, Lorenzo, in: Foro Internacional, Vol. XVI, No. 1, S. 37 ff. (47).

14 Meyer, Lorenzo, in: Comercio Exterior 1975, S. $1334 \mathrm{ff}$. (1340).

$15 \mathrm{Vgl}$. die Wahlergebnisse in: Comercio Exterior 1976, S. $762 \mathrm{ff}$.

16 S. 11 des Manuskripts.

17 Vgl. Jones, Errol D. - Lafrance, David, in: Inter-American Economic Affairs, Vol. XXX (1976), No. 1, S. 45 ff. (60 f.). 
nung der Souveränität der Entwicklungsländer über die eigenen Rohstoffe. (2) Der auf seinen vielen Reisen von Echeverría wahrgenommene Anspruch einer Sprecherrolle in und für die Dritte Welt. (3) Die Inkohärenz der Außenpolitik in spezifischen Konfliktsituationen, in denen (Gebietsansprüche auf Belice ${ }^{18}$, überzogenes Vorgehen gegen Francos Spanien, UN-Zionismusdebatte ${ }^{19}$ ) Mexiko wenig erfolgreich operierte. (4) Rückkehr zum vorsichtigen Taktieren in der Frage der OPEC und der SELA (Sistema Económico Latinoamericano als Zusammenschluß der lateinamerikanischen Länder ohne USA und Kanada, Resolutionen vom August und Oktober 197520). Grabendorff vermutete insoweit eine Rücksichtnahme auf den künftigen Präsidenten Lopez Portillo, der in seiner Politik gegenüber den USA nicht präjudiziert werden sollte.

Leopoldo Solis, z. Z. Princetown University, untersuchte die „Compatibilidad entre el equilibrio externo y el uso pleno de los recursos productivos” zunächst für den "desarrollo estabilizador" der 60er Jahre, gekennzeichnet von den Zielen der Preisstabilität; der Ausgeglichenheit der Zahlungsbilanz, verstanden als Aufrechterhaltung eines festen Wechselkurses (bis zum 30. August 1976), und des wirtschaftlichen Wachstums, erzielt mit dem Instrumentarium der Währungspolitik, der Verschuldung im Ausland und der öffentlichen Ausgaben. Ziele, die Anfang der 70er Jahre nicht eingehalten werden konnten. Die abschließende Frage von Solis, ob angesichts der im Vergleich zu den USA doppelt so hohen Inflationsrate in Mexiko das externe Gleichgewicht mit der historischen Wachstumsrate vereinbar sei, leitete über zu dem Vortrag von Wolfgang König, Universität Göttingen: „Internationale Aspekte der Industrialisierung Mexikos". Angesichts der Verschlechterung der Zahlungsbilanz (in der Höhe der Auslandsverschuldung steht Mexiko nach Indien und Brasilien an 3. Stelle der Entwicklungsländer) und der qualitativen Abhängigkeit vom Investitionsgüterimport sieht König für das kurzfristige Ziel eines Zahlungsbilanzausgleichs als Alternative eine exportorientierte Industrialisierung und Lohnveredelung im grenznahen Bereich zu den USA. In der Diskussion mußte sich König allerdings fragen lassen, ob eine hohe Auslandsverschuldung überhaupt etwas Schlechtes sei, zumal es ökonomisch Grenzen eines Kredits nicht gebe. Den Abschluß des wissenschaftlichen Kolloquiums bildeten zwei dem Einfluß des Tourismus gewidmete Vorträge: Erdmann Gormsen, Universität Mainz, führte unter dem Thema "Tourismus als regionaler Entwicklungsfaktor in Mexiko“ in den unter seiner Leitung an Ort und Stelle eruierten Forschungsgegenstand generell ein, der anschließend von Rüdiger Kreth speziell für Acapulco und die dortige Bevölkerungsstruktur exemplifiziert wurde.

\footnotetext{
$18 \mathrm{Vgl}$. hierzu den Artikel: „Belice: Las amenazas de los militares guatemaltecos ${ }^{\alpha}$, in: Comercio Exterior 1975, S. $1231 \mathrm{ff} .(1234 \mathrm{f}$.).

19 Zum bereits erwähnten dadurch veranlaßten Rücktritt des Außenministers Rabasa: El Heraldo de México v. 31. 12. 1975.

20 Comercio Exterior 1975, S. 1206 ff. und 1211.
} 